\title{
GPPS-BJ-2019-0149
}

\section{TAYLOR-COUETTE-POISEUILLE FLOW HEAT TRANSFER IN A HIGH TAYLOR NUMBER TEST RIG}

\author{
Phillip B. Swann \\ The University of Queensland \\ phillip.swann@uqconnect.edu.au \\ Brisbane, Queensland, Australia
}

\author{
Ingo H. Jahn \\ The University of Queensland \\ i.jahn@uq.edu.au \\ Brisbane, Queensland, Australia
}

\author{
Hugh Russell \\ The University of Queensland \\ h.russell@uq.edu.au \\ Brisbane, Queensland, Australia
}

\begin{abstract}
As technology advances, rotating machinery is becoming smaller and operating at higher rotational speeds, with increased pressure and heat concentration. This combination of factors increases structural stresses, while increasing the risk of temperature sensitive components overheating. To properly protect these components, such as bearings and seals, and reduce structural stresses, it is necessary to have accurately designed thermal management systems with wellunderstood heat transfer characteristics. Currently available heat transfer correlations operating within high Taylor number (above $1 \times 10^{10}$ ) flow regimes are lacking. In this work, the design of a high Taylor number flow experimental test rig is presented. A non-invasive methodology, used to capture the instantaneous heat flux of the rotating body, is presented. A new correlation for Taylor numbers between 0.0 and $9.0 \times 10^{8}$ with air is provided using the effective Reynolds number. Capability of the test rig and methodology enables the use of high density fluids, such as supercritical carbon dioxide, providing opportunity to develop correlations up to $1 \times 10^{12}$. A unique approach is presented, using the MonteCarlo method for evaluating the uncertainties in the calculated values. Data of a single test is presented for a Taylor number of $8.9 \pm 1.6 \times 10^{7}$ and an effective Reynolds number of $3.3 \pm 0.2 \times 10^{4}$. This operating condition corresponded to a measured heat transfer coefficient of $3.16 \pm 0.9 \times 10^{2}$ $\mathrm{W} / \mathrm{m}^{2} \mathrm{~K}$ and Nusselt number of $8.9 \pm 1.6 \times 10^{1}$. This level of detailed uncertainty analysis for heat transfer coefficient measurements is not present in existing literature. This paper represents the first comprehensive portrayal of uncertainty propagation in heat transfer coefficient measurements for Taylor-Couette-Poiseuille (T-C-P) flow heat transfer experiments.
\end{abstract}

\section{INTRODUCTION}

Rotating machinery is used in a wide variety of applications, from turbines and electric generators to motors and workshop equipment. Thermal management has been of increasing interest as machines become smaller and faster (Yin, Tiemstra, and Rao 2018; Heshmat, Ii, and Córdova 2018). High temperature gradients can cause large thermal stresses and shorten the life of temperature sensitive components such as seals, shafts, discs, and bearings. As well as minimizing thermal stresses, minimizing coolant flows is essential to the efficient operation of most rotating machinery. It is therefore critical to have accurate models of the cooling mechanisms present and heat transfer rates to prevent undue stresses and to ensure coolant flow rates are minimized.

Convective heat transfer and fluid dynamics are intrinsically linked, and therefore it is necessary to have a good understanding of the effect of changing fluid regimes on heat transfer rates. Typically, rotating machines involve a rotating shaft with a static outer casing and a small annulus filled with gas or coolant for thermal management and lubrication purposes. The fluid regime which occurs in this mechanical set-up is known as Taylor-Couette (T-C) flow. Adding a mass flow rate in the axial direction results in Taylor-Couette-Poiseuille (T-C-P) flow (Fénot et al. 2011). These flows are described using the Taylor (eq. (1)) and axial Reynolds (eq. (2)) numbers, defined as;

$$
\begin{gathered}
T a=\frac{\rho^{2} \omega^{2} R_{\text {inner }}\left(\frac{D_{h}}{2}\right)^{3}}{\mu^{2}} \\
R e_{a}=\frac{\rho V_{a} D_{h}}{\mu}
\end{gathered}
$$


These non-dimensional numbers describe the flow behaviour within the annulus. The Taylor number is the ratio of centrifugal (rotational) forces to viscous forces, while the axial Reynolds number is the ratio of inertia (axial) forces to viscous forces. In both values, the characteristic length used is the hydraulic diameter of the annulus.

Nusselt number is often presented against an effective Reynolds number, defined in equation (3), as this is thought to capture the axial and rotational components of the flow field within a single value.

$$
R e_{e f f}=\frac{\rho \sqrt{V_{\phi}^{2}+V_{a}^{2}} D_{h}}{\mu}
$$

Many researchers have investigated T-C and T-C-P flow heat transfer, resulting in several review papers (Howey, Childs, and Holmes 2012; P. R. N. Childs and Long 1996; Fénot et al. 2011; Dawood et al. 2015). Although there have been many experimental and numerical studies performed, heat transfer measurements in flows with Taylor numbers above $1 \times 10^{10}$ are lacking.

As research into improving industry operations and equipment continues, high density, low viscosity fluids, such as supercritical carbon dioxide (Heshmat, Ii, and Córdova 2018; Keep, Head, and Jahn 2017), become more prominent and desirable for efficient operation. As these new designs are operating within a higher Taylor number regime, extensions to existing heat transfer correlations, backed by new highquality experimental data, are essential to produce safe and efficient thermal management system designs.

This paper describes the design of a test rig and methodology for measuring T-C-P heat transfer rates in high pressure flows. The maximum design Taylor number for the rig, when operating with supercritical carbon dioxide $\left(\mathrm{sCO}_{2}\right)$ is $1.01 \times 10^{12}$. In the current work, data was gathered with air and a simple correlation developed over the Taylor number range of $7.4 \times 10^{6}$ to $6.6 \times 10^{8}$. This new correlation is discussed and compared with existing correlations within this Taylor number range.

The paper is structured as follows. First, a short review of previous experiments to characterise T-C-P flows is presented. This is followed by a description of the test rig design, including CFD simulations to show the flow structure within the test region. Then a description of the methodology detailing the calculation of the heat transfer coefficient and uncertainty quantification using the Monte-Carlo method is presented. Lastly, a first set of data are analysed, and a new correlation for air is presented.

\section{LITERATURE REVIEW}

Many researchers have performed experiments to determine both flow transition stages (Smith and Townsend 1982; Masuda et al. 2018) and heat transfer characteristics (Aoki, Nohira, and Arai 1967; Ball, Farouk, and Dixit 1989) for T-C and T-C-P flow regimes. To date, the most extensive range of heat transfer data for T-C-P flows has been compiled by Fénot et al. (2011) which spans a range of $\mathrm{Ta}=0$ to $1.2 \times 10^{11}$ and $R e_{a}=2.93 \times 10^{2}$ to $1.37 \times 10^{6}$. In some industrial applications, supercritical carbon dioxide turbine design, for example, Taylor numbers greater than $1 \times 10^{11}$ may be encountered. As T-C-P flows are inherently unsteady and three dimensional, using high fidelity CFD simulations (e.g. LES or DNS) are not tenable. Hence, gathering new experimental data at these flow conditions is necessary.

Experimental heat transfer data gathered for the highest Taylor number range (up to $1.2 \times 10^{11}$ ) is currently by Childs and Turner (1994). Their experimental set-up used a $0.4 \mathrm{~m}$ diameter rotor, rotated at speeds up to 10000 RPM and a mass flow of $4 \mathrm{~kg} . \mathrm{s}^{-1}$. This arrangement gave Taylor numbers in the range 0 to $1.2 \times 10^{11}$ due to the rotor's high surface speeds. A large ducted fan system was used to draw air at atmospheric conditions through an initial non-rotating zone, used to ensure fully developed flow at the test section. (P. Childs and Turner 1994).

The large diameter rotor used in the design by Childs and Turner (1994) prevented the use of high pressure and density flows. Higher pressures at these diameters would require a prohibitively thick outer casing to ensure safe operation. The design also did not allow for alternate fluids at varying conditions to be used. This limited the test range of fluid properties.

A critical aspect of establishing heat transfer relations is the measurement of heat transfer to the rotating shaft. Childs and Turner (1994) achieved this using embedded heat transfer gauges, but this is limited as the operating conditions of the test rig must be within the operating limits of the sensors. A more extensive study of measurement techniques was performed by Seghir-Ouali et al. (2006) who determined the convective heat transfer coefficient for air passing through a rotating cylinder, known as Hagen-Poiseuille flow. Their setup consisted of power regulated infrared heaters to heat the test section and an infrared camera to measure the temperature history of the shaft surface. The team determined the convective heat transfer using three different methods; a steady state inverse discretized model, a thermally thin wall assumption method, and an analytical method (Seghir-Ouali et al. 2006). All three methods were found to be of similar relative uncertainty in the final heat transfer coefficient calculation. Key advantages of the heat transfer measurement techniques employed by Seghir-Quali et al. (2006) are that they are non-invasive, and instrumentation can be kept outside of the test rig, removing constraints on operating temperature, speed, and pressure.

A further evolution of heat transfer measurement techniques using system inversion for transient temperature changes is presented by Battaglia et al. (2001). The method presented assumes that the thermal system is linear and thus temperature response is the convolution of the heat flux with the impulse response of the system in question. Thus, by identifying the system response and de-convolving the temperature history, the transient input of heat flux can be recovered. This process was applied by Battaglia et al. (2001) to determine the heat flux between a cutting tool and material. The identified system was used to estimate both temperature and heat flux on the cutting surface. It was found that the calculated temperature profile from the convolution of heat 
flux and impulse response of the cutting edge was 'very close' to the experimentally measured temperature. Another study was performed on null point calorimeters in high speed plasma flow characterization using the same method (Gardarein, Battaglia, and Löhle 2009). It was found that the method performed better than other established experimental approaches, due to the slow response from the thermal inertia of the sensors used in the other experiments.

One of the key advantages of this approach is that it does not assume a heat input function (e.g. step function) and calculates an instantaneous heat input at each time interval. The method also does not require any knowledge of fluid properties or behaviour to determine the heat transfer rate. A variation of the method described by Battaglia et al. (2001) is used in this body of work.

\section{TEST RIG}

A picture of the test rig is shown in Figure 1, and a schematic representation displayed in Figure 2. The rig operates transiently whereby it is initially heated. Then flow is initiated to cool the test section. With this approach, the initial temperature of the test rig is uniform and can be measured. As data is collected in the transient phase, before the effects of cooling are propagated to the outside of the casing, this simplifies the outer thermal boundary conditions for the heat transfer analysis.

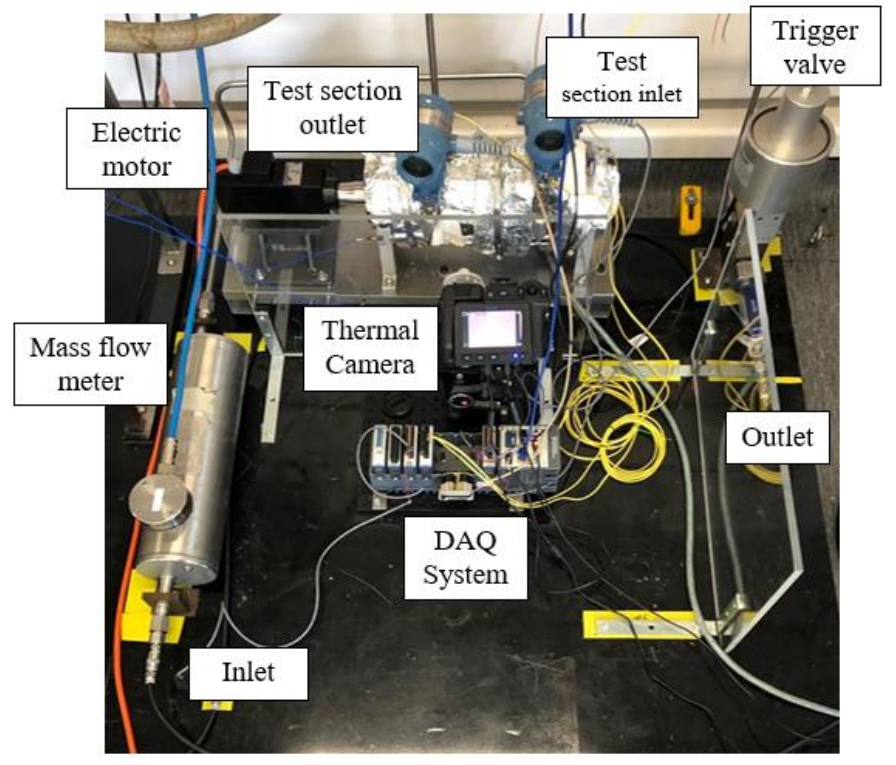

Figure 1 Experimental test rig showing component assembly and layout

The test section consists of an inlet and outlet plenum with a long annular section between them. The long annular section will be referred to as the test region. The test region is designed to be long enough for flow to be fully developed before the flow reaches the optical windows. A cross-section of the test section is shown in Figure 2.

The test rig has a $10 \mathrm{MPa}$ and $150^{\circ} \mathrm{C}$ operating pressure and temperature limit, providing the ability to test high density and supercritical fluids. An electric motor is used to spin the shaft up to 25000 RPM. The combination of high density, low

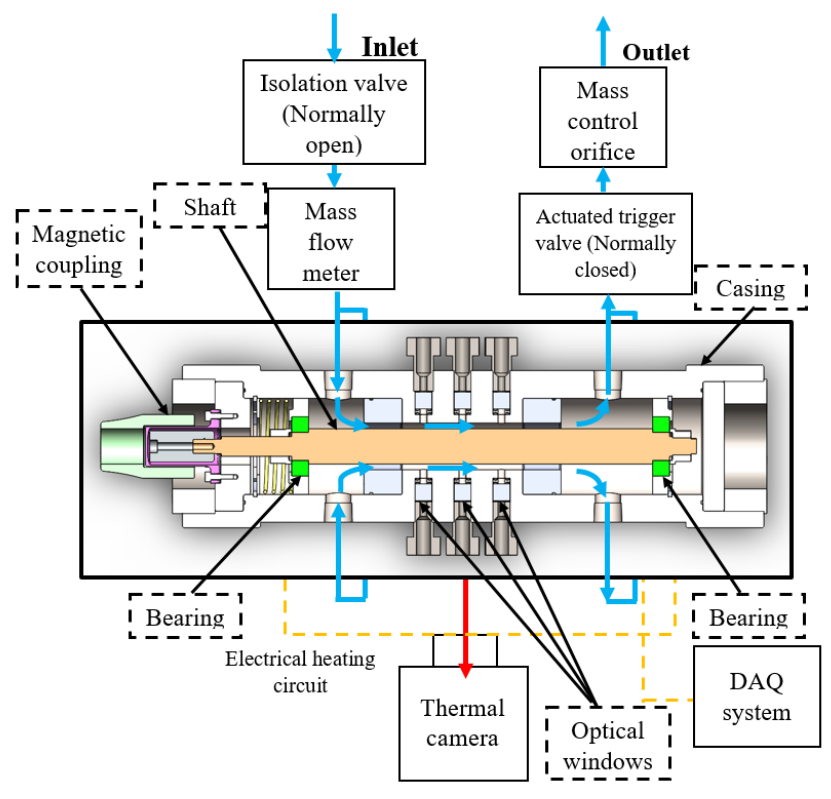

Figure 2 Test rig layout schematic

viscosity fluids, and shaft speed, enables Taylor numbers up to $1.01 \times 10^{12}$ to be achieved. The axial mass flow rate through the test section is controlled by an orifice plate placed before the rig outlet. Mass flow rate can be varied from 0.0 to $0.01 \mathrm{~kg} / \mathrm{s}$, giving an axial Reynolds number range of 0 to $1.84 \times 10^{5}$. These design aspects provide critical control over the rotational and axial components of fluid flow, which allows the interdependency between flow and overall heat transfer to be accurately characterised. The key parameters of the test rig are summarised in Table 1.

Table 1 Key parameters of test rig design

\begin{tabular}{lcc}
\hline \hline & $\begin{array}{c}\text { Maximum (or } \\
\text { Nominal) }\end{array}$ & Minimum \\
\hline Shaft diameter $(\mathrm{mm})$ & 25 & - \\
Annulus height $(\mathrm{mm})$ & 4 & - \\
Test region length $(\mathrm{mm})$ & 136 & - \\
Shaft speed $(\mathrm{RPM})$ & 25000 & 0 \\
Pressure $(\mathrm{MPa})$ & 10 & $\geq$ ambient \\
Mass flow rate $(\mathrm{kg} / \mathrm{s})$ & 0.01 & 0 \\
Temperature $\left({ }^{\circ} \mathrm{C}\right)$ & 150 & $\geq$ ambient \\
Shaft surface speed $(\mathrm{m} / \mathrm{s})$ & 32.7 & 0 \\
Taylor number & $1.01 \times 10^{12}$ & 0 \\
Axial Reynolds number & $1.84 \times 10^{5}$ & 0 \\
Test duration $(\mathrm{s})$ & $15-60$ & - \\
\hline \hline
\end{tabular}

To avoid the need for seals, the shaft is connected to a Perske VS31.09 electric motor via a magnetic coupling manufactured by DST Magnetic Couplings. The pressure vessel (and shaft) are heated using $4 \mathrm{~m}$ of 50PHT Heat Trace cable connected to an RS PRO 48x48 temperature controller unit. The controller thermocouple is placed on the outside of the casing, shown in Figure 2.

\section{Test Procedure}

The operating procedure of the test rig is to first bring the test section to temperature, while the shaft is stationary and the 
test section is at low pressure. Once the shaft and casing has reached uniform and steady temperature, the upstream isolation valve is opened, and the test section is pressurised. Once the introduced gas has reached temperature and the temperature of all components in the test section has equilibrated, tests commence. During a test, first the shaft is brought to operating speed and then the trigger valve (see Figure 2) is opened. This allows the hot gas within the rig to escape and a cold slug of gas originally upstream of the mass flow meter to flow through the test rig. The test-time starts when this cold slug reaches the test section. Each test ends by closing the trigger valve. During tests, the mass flow rate and pressure inside the rig is controlled by the orifice plate positioned at the downstream end of the test section and the supply pressure.

The transient nature of the tests, and in particular the cold gas slug arriving in the hot test section allows an analytical inverse deconvolution technique to be used in determining the convective heat transfer from the shaft surface temperature history. Shaft surface temperature is measured using a thermal camera as described in more detail later.

\section{Sensors and Data Acquisition}

The inlet and outlet plenums of the test section include Rosemount 2051 pressure transmitters and K-type thermocouples. A third thermocouple was positioned radially in the centre of the annular flow, axially at the position of the surface temperature measurement. This was used to provide a reference temperature for $\mathrm{T}_{\mathrm{f}}$. For tests with air, the downstream orifice plate was used to calculate mass flow through the rig. Annex A of the International Standard ISO 5167 was used to evaluate the mass flow rate. The outlet plenum measurements were used for as the upstream conditions of the orifice plate with atmospheric conditions for the downstream. For operating with higher density fluids, the Siemens SITRANS FC MASS 2100-DI15 coriolis type mass flow meter is available. The pressure, temperature, and mass flow rate measurements are routed through a Compact-RIO NI 9072 chassis and a custom LabVIEW data acquisition program to obtain the measurement data, which is sampled at $200 \mathrm{~Hz}$.

The accuracy of the K-type thermocouples is $\pm 2.2 \mathrm{~K}$, and the Rosemount pressure transmitters have an accuracy of $\pm 0.026 \mathrm{MPa}$. The coriolis mass flow meter has an accuracy of $\pm 0.15 \%$ at the mass flow rates used in testing. These accuracies were taken from the data sheets provided with the sensors. Thermocouple and pressure transmitter calibrations were conducted. A $95 \%$ confidence interval in the uncertainties over the range of temperatures and pressures of testing were found to be $\pm 3.6 \mathrm{~K}$ and $0.026 \mathrm{MPa}$, respectively. The calibration uncertainty is used in the uncertainty quantification section as it is larger than those from the data sheets.

Aremco 840M high emissivity paint with an emissivity of $0.95 \pm 0.05$ is used on the shaft surface for a high infrared signal to be received by the FLIR T650sc thermal imaging camera. The thermal camera is routed directly to a PC and is operated using the FLIR ResearchIR MAX software. The data collected using the software is amalgamated and analysed using a Python script.

\section{Simulation of Test Section}

To understand the flow structures present within the test region, to confirm that end-effects are negligible, and to show that correct flow structures expected from T-C-P flow are established in the test region, a 3D Computational Fluid Dynamics (CFD) analysis was performed. By understanding these aspects, it has assisted in interpreting the heat transfer data gathered.

\section{Simulation Setup}

The ANSYS CFX CFD solver was used to perform the analysis. A schematic of the simulation domain is displayed in Figure 3, showing where the domain boundaries are defined. A mass flow inlet was defined on the face of the tube stub section, located at the entrance to the inlet plenum. A pressure outlet was defined at the outlet plenum in a similar manner. A rotating, constant temperature wall was defined for the inner boundary of the fluid domain (shaft surface). The outer boundary was set to a stationary wall at constant temperature (inner casing surface). The first cell height was set to a y+ value of approximately 1 to ensure the boundary layer is appropriately resolved, resulting in a radial cell height of $3.5 \times 10^{-6} \mathrm{~m}$ at the walls, when using air as the domain fluid.

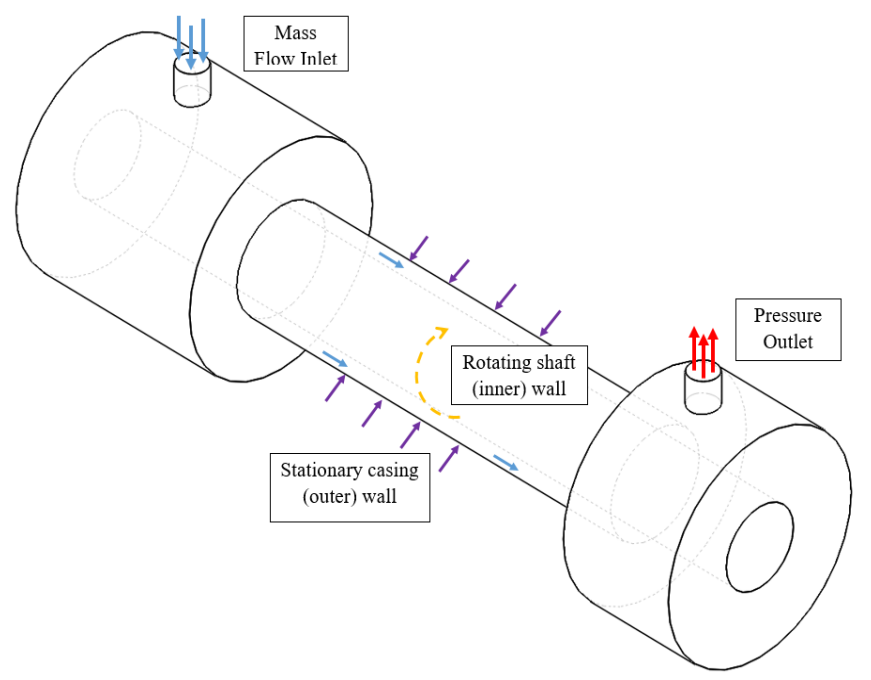

Figure 3 Schematic of CFD flow domain

Both steady and unsteady RANS simulations were conducted for the nominal operating point. These provided similar results for heat transfer and flow structures in the test region. Due to the good agreement and the reduced cost of the steady RANS simulation, these were used for extensive analysis of the test rig and are presented in this publication. For these steady state simulations, fixed wall temperatures were prescribed on the inner and outer wall. This approach allows the simulation of a snap-shot of the flow part-way through the experimental test time.

The momentum equations were closed with the k- $\omega$ SST turbulence model. As the flow is incompressible $(\mathrm{M}<0.3)$, the equations were solved using the SIMPLE algorithm including 
the energy equation to account for temperature and density variations. Boundary conditions for the CFD simulation are displayed in Table 2.

Table 2 Conditions set for CFD study

\begin{tabular}{lcc}
\hline \hline Boundary & Type & Value \\
\hline \multirow{2}{*}{ Inlet } & Mass flow & $0.003 \mathrm{~kg} / \mathrm{s}$ \\
& Temperature & $40^{\circ} \mathrm{C}$ \\
Outlet & Pressure & $0.28 \mathrm{MPa}$ \\
\multirow{2}{*}{ Shaft wall } & Moving wall & $20000 \mathrm{RPM}$ \\
& Temperature & $75^{\circ} \mathrm{C}$ \\
Casing wall & Stationary wall & N/A \\
& Temperature & $90^{\circ} \mathrm{C}$ \\
\hline \hline
\end{tabular}

\section{Verification}

A verification study was performed to determine the suitability of the boundary conditions and mesh refinement. To simplify the flow problem, pure Taylor-Couette (T-C) flow (no axial mass flow) was used in the verification study. For this verification study the inlet boundary was set to a pressure inlet and the outlet to a stationary wall at constant temperature.

Figure 4 shows the heat transfer coefficient (HTC) for the centre of the test region sufficiently far from the shaft ends to eliminate end effects, for three increasing refinements to the mesh. The heat transfer coefficient is calculated using the wall heat flux at the inner wall and the difference between the inner and outer wall temperature. The average over the test region is also displayed. As the axial placement of the vortices shifts between the runs, the raw HTC values for Ref \#2 has been shifted axially to better illustrate agreement between T-C flow structures and corresponding HTC profiles.

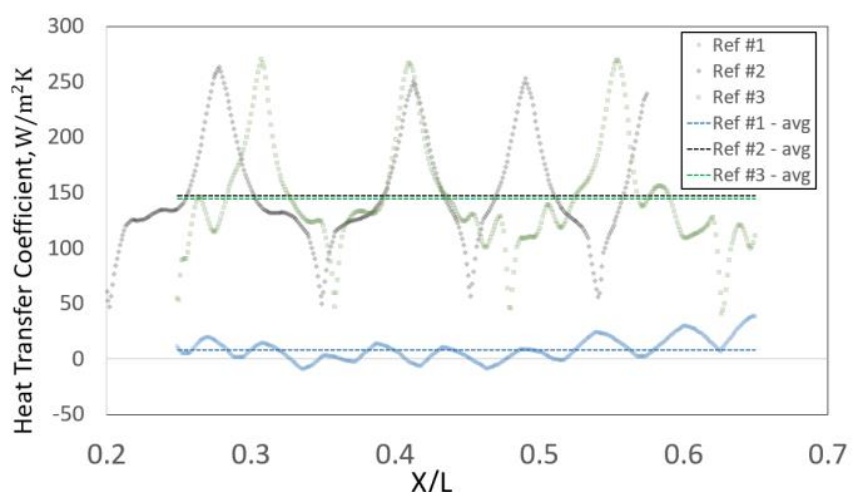

Figure 4 Spatial variation of HTC for mesh refinement study for pure T-C flow

The mesh sensitivity study shows a converging average HTC for increasing mesh refinements as also reported in Table 3. For the two finer meshes, $6.2 \times 10^{6}$ and $8.7 \times 10^{6}$ cells, the relative error in average HTC has reduced to $1.8 \%$, which is sufficient for the current CFD study. These HTC results were compared to literature for the relevant Taylor number $\left(T a=1.3 \times 10^{8}\right)$. Using the correlation developed by Tachibana et al. (1960), the analytically obtained HTC for the simulation conditions is $142 \mathrm{~W} / \mathrm{m}^{2} \mathrm{~K}$, which is within $3.7 \%$ and $1.9 \%$ for predicted values for Ref \#2 and Ref \#3. For further simulations, a mesh with a refinement similar to midpoint between Ref \#2 and \#3 and extended inlet and outlet plenums was selected.
Table 3: Dependency study results

\begin{tabular}{lccc}
\hline \hline Parameter & Ref \#1 & Ref \#2 & Ref \#3 \\
\hline Cells & $4.98 \times 10^{6}$ & $6.22 \times 10^{6}$ & $8.70 \times 10^{6}$ \\
$\begin{array}{l}\text { Average HTC } \\
\left(\mathrm{W} / \mathrm{m}^{2} \mathrm{~K}\right)\end{array}$ & 8.45 & 147.3 & 144.7 \\
$\begin{array}{l}\% \text { Error } \\
\text { relative Ref \#3 }\end{array}$ & 94.1 & 1.8 & $\mathrm{n} / \mathrm{a}$ \\
\hline \hline
\end{tabular}

\section{CFD Results}

To provide insight into the operation of the rig, T-C-P flow with boundary conditions prescribed in Table 2 was simulated. Figure 5 shows the corresponding results as temperature contours on an axial slice through the test section with overlaid velocity vectors. From these results, clear Taylor vortices and the corresponding localised enhancement in heat transfer by transport of hot fluid to the shaft are evident in the latter half of the test section. The 'Stabilised T-C-P' region was identified by the presence of a regular helical flow structure and the point where the mid annulus temperature started to show a linear increase.

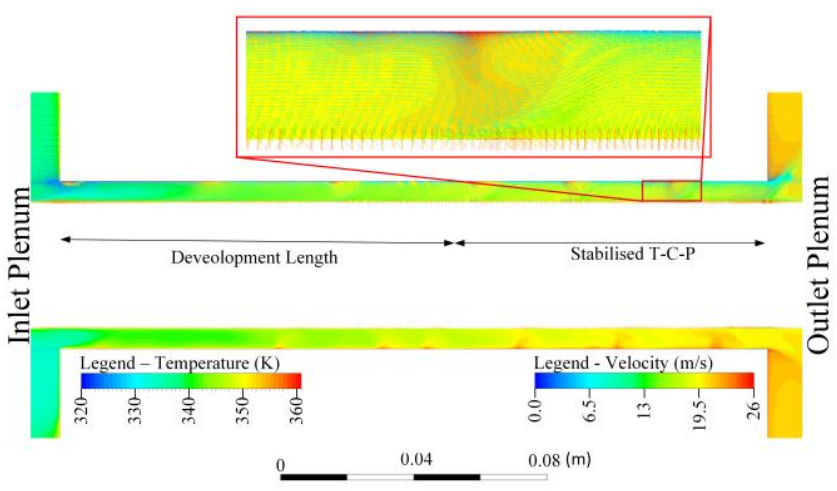

Figure 5 CFD results for T-C-P flow in test region

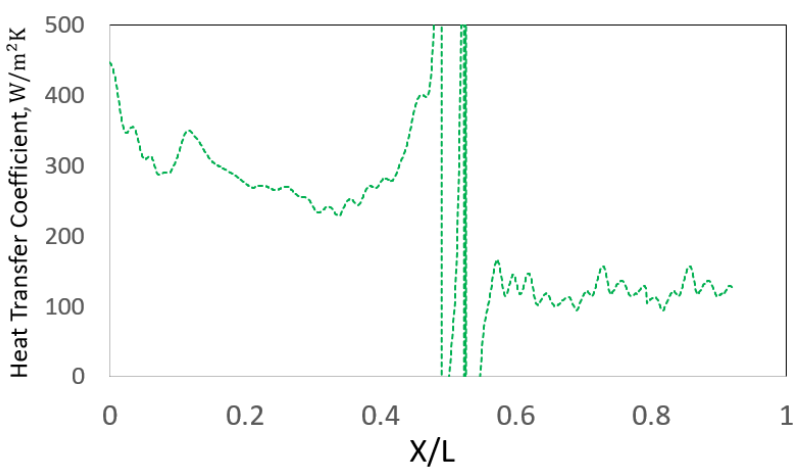

Figure 6 Shaft surface Heat transfer coefficient for T-C-P flow case (test region starts at $X / L=0$ )

Figure 6 displays the corresponding HTC profile along the shaft surface. HTC is calculated using the inner wall heat flux and the difference between the inner wall temperature and averaged temperature taken at the centre of the annulus. The averaged mid channel temperature is obtained through a linear regression fit to the mid channel temperature in the 'Stabilised T-C-P' region. HTC was calculated along four lines spanning the length of the test region and placed equidistant around the 
inner wall of the flow domain. The HTC values were then circumferentially averaged, resulting in Figure 6.

Figure 6 shows a decreasing HTC up to $X / L=0.4$. There is a significant fluctuation in HTC between $X / L=0.4$ and 0.6. This is caused by the temperature difference between the mid annulus and shaft surface approaching zero. After $X / L=0.6$, the HTC value stabilises. The stabilization of HTC matches the region of established Taylor vortices ("Stabilised T-C-P" in Figure 5).

These simulations confirm that Taylor vortices form inside the test rig anulus and that by the measurement location used for the experiments, positioned at $X / L=0.65$ from the test region start, stabilised T-C-P flow exists. The simulation conditions from Table 2, correspond to the highest axial flow velocity of the experimental cases, giving a conservative estimate of the development length.

\section{METHODOLOGY}

This section will describe the necessary steps to determine the Heat Transfer Coefficient (HTC) from the measurements taken in the test rig. The determination of the HTC is a three-stage process. Each stage is described in the subsequent sections.

\section{Measurement of Surface Temperature}

The measurement of the shaft surface temperature is a two-stage process. First, the FLIR T650sc thermal camera is used to measure the thermal signal coming from the shaft surface. This produces the raw camera signal as counts. Second, the camera counts measurements are converted into temperature measurements via a two-point calibration curve that is established before each series of tests and verified afterwards.

As the test rig does not include instrumentation for direct measurement of the shaft surface temperature, $\mathrm{T}_{\mathrm{s}}$, this has to be estimated from other thermocouples in the rig to allow for accurate calibration. A series of off-line calibration experiments were conducted that included an invasive thermocouple attached directly to the shaft. From these, it was established that the shaft surface temperature, $\mathrm{T}_{\mathrm{s}}$, used in the calibration process could be accurately estimated from the fluid reference temperatures taken in the inlet and outer plenums. The uncertainty associated with the approach is included as a calibration uncertainty in the later error propagation study.

An individual calibration curve is developed for the series of tests to eliminate any discrepancy in measurement caused by change in camera focus or atmospheric conditions. This insitu calibration also accounts for the presence of lenses, optical windows, and the gas path between the window and shaft.

The relationship between the two points used in the calibration curve was shown to be linear in a series of previous experiments. These experiments, not covered in this paper, were performed to investigate the effect of the possible interferences in the path of the camera signal. These included the fluid used within the test rig (between the shaft surface and the optical window), the optical window, and the effect of heating the test rig during testing. It was shown that these have a consistent impact and that their influences can be removed through the two-point calibration process.

\section{Calculation of Surface Heat Flux}

From an energy balance point of view, the total heat transfer between the rotating shaft and cooling fluid comprises of conduction, convection, and radiation components. Due to the low temperature of the rig, the radiation component is sufficiently small so that it can be ignored without loss of accuracy. The conduction and convection components manifest as heat transfer from shaft to fluid, which is balanced by conduction within the shaft.

Previous works have shown that the temperature of a linear system can be represented as the convolution of the heat input to the system and the systems impulse response (Battaglia et al. 2001). Converting the analytical equations for the shaft and fluid heat transfer into discrete form yields the following linear relationship between discrete heat flux and temperature values.

$$
\boldsymbol{T}=\boldsymbol{I} \boldsymbol{Q}=\left[\begin{array}{c}
T_{0} \\
T_{1} \\
\vdots \\
T_{N}
\end{array}\right]=\left[\begin{array}{cccc}
I_{0} & 0 & \cdots & 0 \\
I_{1} & I_{0} & 0 & \vdots \\
\vdots & \vdots & \ddots & 0 \\
I_{N} & I_{N-1} & \cdots & I_{0}
\end{array}\right]\left[\begin{array}{c}
q_{0} \\
q_{1} \\
\vdots \\
q_{N}
\end{array}\right]
$$

Therefore, taking the discrete measured temperature history $T_{0}$ to $T_{N}$ and applying the system impulse response $I_{0}$ to $\mathrm{I}_{\mathrm{N}}$ derived analytically, the corresponding discrete time history of the heat flux, $q_{0}$ to $q_{N}$, can be determined by taking the inverse of the impulse response matrix.

$$
Q=I^{-1} T
$$

To apply this method, firstly, it is necessary to define the governing equation for transient conduction heat transfer with relevant boundary and initial conditions. As the shaft has a high rotational speed, it can be assumed that any circumferential variations in heat transfer as experienced by the shaft surface are smeared out, and the circumferential component of the conduction equation inside the shaft is sufficiently small to be neglected. The axial component can also be neglected as the shaft starts off at a uniform temperature, and heat transfer only varies slowly in the axial direction, which means that during the initial transient phase, shaft temperatures are dominated by heat transfer in the radial direction.

From these assumptions, the governing equation is the 1$\mathrm{D}$ (radial component) transient conduction heat equation in cylindrical coordinates shown in eq. (6). Corresponding boundary conditions and initial conditions are listed in equations (7) to (9).

$$
\begin{gathered}
\frac{1}{\alpha} \frac{d T}{d t}=\frac{1}{r} \frac{d}{d r}\left(r \frac{d T}{d r}\right) \\
-\left.k \frac{d T}{d r}\right|_{r=0}=0 \\
-\left.k \frac{d T}{d r}\right|_{r=R}=-q \\
T(r, 0)=0
\end{gathered}
$$

Solving the governing equation using an auxiliary function and the separation of variables approach, leads to the 
temperature equation for a step response to input heat flux with magnitude q:

$$
\begin{gathered}
T(r, t)=q\left(\frac{r^{2}}{2 k R}+\frac{2 \alpha t}{k R}\right)+\sum_{n=0}^{\infty} C_{n} J_{0}\left(\lambda_{n} r\right) e^{-\lambda_{n}^{2} \alpha t} \\
C_{n}=\frac{\int_{0}^{R} r\left(T_{\text {init }}-\frac{q r^{2}}{2 k R}\right) J_{0}\left(\lambda_{n} r\right) d r}{\int_{0}^{R} r J_{0}\left(\lambda_{n} r\right)^{2} d r} \\
J_{1}(\lambda R)=0
\end{gathered}
$$

Where $J_{0}$ and $J_{1}$ are the Zeroth and First Order Bessel functions of the first kind, respectively. Looking at eq. (10) and (11), the functions are linear with respect to the heat flux for the case that initial temperature, $T_{\text {init }}=0$. This can be achieved by offsetting the discrete measured temperature series with the temperature at time, $t=0$. The linearity is a necessary characteristic of the system in order to use the convolution of heat input and impulse response. The impulse response of the system is found by taking the derivative of the analytical temperature equation (eq. (13)).

$$
\frac{d T(r, t)}{d t}=q\left(\frac{2 \alpha}{k R}\right)-\sum_{n=0}^{\infty} \lambda_{n}^{2} \alpha C_{n} J_{0}\left(\lambda_{n} r\right) e^{-\lambda_{n}^{2} \alpha t}
$$

The impulse matrix is formulated using the time resolution of the temperature measurements. Using the impulse matrix and the temperature history obtained from the thermal camera measurements, the discrete heat flux vector can be determined through elementary matrix operations as defined in eq. (5).

\section{Calculate HTC}

From the heat flux vector, the heat transfer coefficient (HTC) is determined through Newton's law of cooling for all time intervals.

$$
h_{i}=\frac{q_{i}}{\left(T_{s i}-T_{f i}\right)}
$$

This equation uses the estimated fluid temperature, $T_{f}$, and the shaft surface temperature, $T_{s}$, measured using the thermal camera. $h_{i}$ is the instantaneous heat transfer coefficient (HTC) at time interval $i$. The temperature of the fluid at time interval $i\left(T_{f i}\right)$ is determined in two ways. First, if the middle thermocouple positioned at the surface measurement location is present for a test, this measurement is used directly as $T_{f i}$. If this thermocouple was not present for the test, it is estimated by assuming a linear temperature profile between the inlet and outlet plenums to calculate the fluid temperature at the measurement location.

To determine the HTC for a given test, the cumulative average of the HTC values in the time series is determined. This is evaluated by averaging the HTC values up to each time step, producing a smoothed HTC curve. This provides the necessary clarity to determine when the HTC value has stabilised. The final HTC value is then evaluated by averaging the raw HTC values over the stabilised region.

\section{Uncertainty Quantification}

The detailed analysis of the uncertainty propagation and corresponding quantification is split into three steps: (1) Establish uncertainty in shaft surface temperature, (2) establish uncertainty in surface heat flux q, and (3) establish uncertainty in the HTC.

\section{Shaft Surface Temperature Uncertainty}

Shaft surface temperature is measured using a calibrated IR camera. The calibration is performed using a linear curvefit relating the raw camera counts to surface temperature. This calibration process accounts for the as-built optical losses and is performed against a $\mathrm{K}$-type reference thermocouple (used to infer shaft temperature during calibration). The calibration curve is then offset to account for the difference in shaft and fluid temperature at the time of the daily calibration. This optical temperature measurement approach introduces a systematic uncertainty of $\pm 4.9 \mathrm{~K}$. In addition, there is a random uncertainty of $\pm 0.3 \mathrm{~K}$ when taking IR measurements.

\section{Surface Heat Flux Uncertainty}

Surface heat flux, $q_{0}$ to $q_{N}$, is calculated using eq. (5), which is reliant on a series of discrete temperatures, $T_{0}$ to $T_{N}$. To propagate uncertainties through this process and to capture the impact of shaft properties and geometry, the Monte Carlo method is applied. For this, 1000 different temperature traces are created by first adding the same normally distributed systematic uncertainty to all temperature data points, and then adding normally distributed random uncertainties to each individual data point. This approach ensures consistent systematic errors are used for each discrete temperature series. For each of the 1000 different temperature traces, shaft geometry and properties are randomly selected based on square or normal distributions. Properties and uncertainties are displayed in Table 4.

Table 4 Shaft property uncertainties and distributions

\begin{tabular}{lcc}
\hline \hline Property & Uncertainty ( \pm ) & Distribution \\
\hline Radius $(\mathrm{m})$ & 0.00025 & square \\
Density $\left(\mathrm{kg} / \mathrm{m}^{3}\right)$ & 80 & normal \\
Heat capacity $\left(\mathrm{J} / \mathrm{kgK}^{-1}\right)$ & 50 & normal \\
$\begin{array}{l}\text { Conductive heat } \\
\text { transfer coefficient }\end{array}$ & 1.6 & \\
$\left(\mathrm{~W} / \mathrm{mK}^{-1}\right)$ & & normal \\
\hline
\end{tabular}

Heat flux traces are calculated for each of the 1000 temperature traces to generate 1000 different heat flux traces. The uncertainty for heat flux, q, is then established by taking the mean of all the heat flux traces obtained from the MonteCarlo analysis and determining the $95 \%$ confidence intervals for each discrete point in time.

Results from the Monte-Carlo analysis using 900 and 1000 temperature traces were compared, giving a 95\% confidence interval of $\pm 3.3 \times 10^{1}$ and $\pm 3.5 \times 10^{1}$ respectively. As the difference in confidence interval is small, it was concluded that using 1000 temperature traces is sufficient to capture the uncertainty limits.

\section{HTC Uncertainty}

The heat transfer coefficient (HTC) uncertainty is calculated using a further Monte Carlo simulation of equation (14). In 
this equation, HTC is dependent on the heat flux, surface and fluid temperatures. The temperature measurements for the inlet and outlet plenums and middle thermocouple, used to calculate $T_{f i}$ were calculated to be normally distributed. HTC is then calculated at each time interval for each Monte-Carlo trace of heat flux and corresponding temperatures. The uncertainty in HTC is established by taking the $95 \%$ confidence interval of all the HTC traces over the stabilised region from which the final HTC value was determined.

\section{RESULTS AND DISCUSSION}

Results for a single test with nominal conditions described in Table 5 are shown in Figure 7. Displayed in Figure 7 (Top) are the measured camera counts, the calculated shaft surface temperature and air temperatures at the inlet and outlet plenums. Before the test begins, there is a constant temperature of $\mathrm{T}=76^{\circ} \mathrm{C}$ followed by a short rise to $\mathrm{T}=77^{\circ} \mathrm{C}$. The test starts as the trigger valve is opened $(\mathrm{t}=20.6 \mathrm{~s})$ and the cooling air passes through the test section. Coincident with the pressure reduction, indicating flow, temperature begins to decrease $(t=21.0 s)$. The air temperature shows a difference
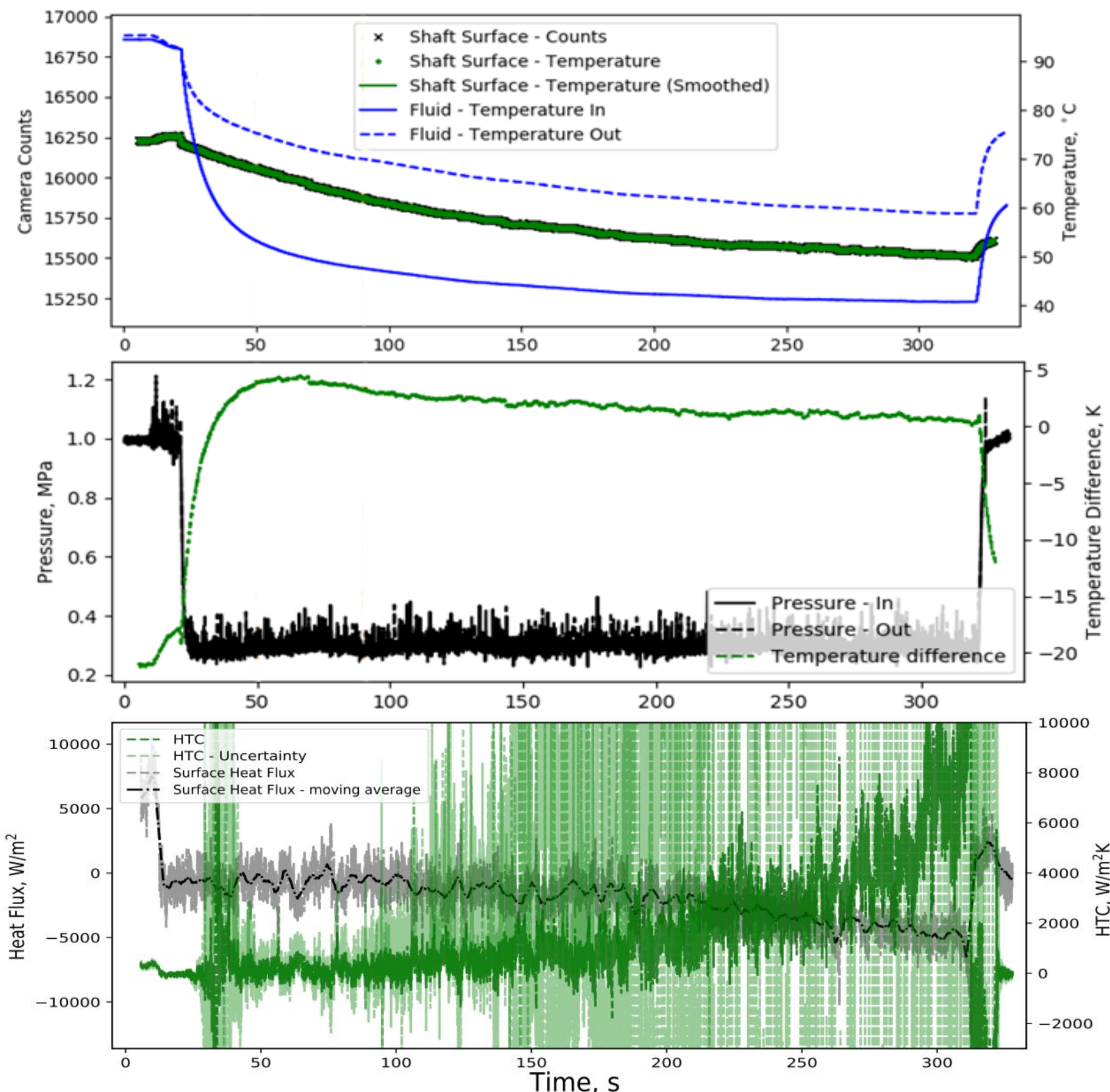

Figure 7 (Top) Surface and Fluid Temperature; (Middle) Air Pressure and Shaft-Fluid Temperature Difference; (Bottom) Heat flux and HTC 
after $\mathrm{t}=21.7 \mathrm{~s}$, between the inlet and outlet plenums. This difference indicates that there is heat transfer to the flow as expected and is attributed to the transfer between the shaft, fluid and casing.

Table 5 Nominal test conditions for demonstration

\begin{tabular}{lc}
\hline \hline Parameter & Value \\
\hline Speed $(\mathrm{RPM})$ & 20000 \\
Fluid Inlet Temperature $\left({ }^{\circ} \mathrm{C}\right)$ & 40 \\
Fluid Inlet Pressure $(\mathrm{MPa})$ & 1.0 \\
Mass control orifice size $(\mathrm{mm})$ & 8 \\
Test time $(\mathrm{s})$ & 300 \\
Shaft Initial Temperature $\left({ }^{\circ} \mathrm{C}\right)$ & 90 \\
Nominal Taylor Number & $9 \times 10^{7}$ \\
Nominal Axial Reynolds Number & $3.4 \times 10^{3}$ \\
\hline \hline
\end{tabular}

A large temperature difference is present between the fluid and shaft surface at the start of the test before the trigger valve is opened $(t=0 s)$. This is due to the test starting before the shaft reached a thermally equilibrated state with the fluid. The system inversion, used to determine heat flux from the shaft surface temperature, assumes the shaft initially has a constant radial temperature distribution. As the conductive heat transfer coefficient within the shaft material is significantly greater than the convective heat transfer to stationary air (i.e. the system has a small Biot number), the effect of the initial temperature difference on the radial temperature distribution is expected to be small.

The shaft and fluid are only required to be in thermal equilibrium during the daily camera calibration, as the fluid temperature is used as a proxy for the shaft surface temperature. After the initial calibration, large temperature differences may be present without effecting the calculation of the heat flux or HTC. Subsequent tests throughout the day only require the measurement of heat flux, fluid and shaft surface temperature and a stabilised region over which to provide the final HTC value for those test conditions. This indicates that the presence of an initial temperature difference does not falsify the data collected at these test conditions.

Figure 7 (Middle) displays the air pressure through the test region and the temperature difference between the shaft surface and averaged fluid temperature, used to calculate the HTC. The reduction in pressure, from $0.99 \mathrm{MPa}$ to $0.28 \mathrm{MPa}$, at the start of the test $(\mathrm{t}=20.8 \mathrm{~s})$, air venting through the orifice

The pressure magnitudes measured up and downstream of the test region are indiscernible. After an initial start-up lasting approximately 4.1 seconds, pressure stabilises at $0.28 \mathrm{MPa}$.

A significant increase in fluctuation in the pressure measurement (increasing from $\pm 0.03 \mathrm{MPa}$ to $\pm 0.3 \mathrm{MPa}$ ), is observed at $\mathrm{t}=10.8 \mathrm{~s}$, the point in time when the motor is started. Further analysis is still underway to establish the cause of the increase in fluctuation.

A region up to $t=50$ s, shows a quickly changing temperature difference between shaft surface and fluid, where the cold gas slug enters the test section. After this initial change, a slow decrease from 4.9 to $1.2 \mathrm{~K}$ difference is seen over the rest of the test time. This indicates there is significant changes in heat transfer or heat transfer coefficient over the transient period.
Figure 7 (Bottom) shows the heat flux and HTC. It is observed that the process to de-convolve the discrete time history amplifies measurement noise. The raw heat flux is indicated in grey. A moving average is performed on the heat flux to reduce the amplified noise, indicated in black. The HTC is calculated using the raw heat flux value and is shown in dark green. The uncertainty on the calculated HTC value is indicated in light green. The calculated HTC shown in Figure 7 , uses an estimated value for the fluid temperature, $T_{\mathrm{fi}}$, based on a linear interpolation between inlet and outlet fluid temperature as the extra fluid thermocouple was not yet installed.

A significant fluctuation in HTC is present in Figure 7 (Bottom) at $\mathrm{t}=29.5 \mathrm{~s}$ with large uncertainties. This fluctuation corresponds to when the temperature difference between the shaft surface and fluid temperature is near zero, seen in Figure 7 (Middle), when the initial cold gas slug enters the test section. As the temperature difference approaches zero, the HTC approaches infinity causing these fluctuations. This is followed by a region of steady heat transfer, lasting until $\mathrm{t}=$ 90s for the data shown in Figure 7. Thereafter, HTC is seen to increase steadily, and uncertainties increase again as the temperature difference reduces.

This steady region at the beginning of a test is used to establish HTC for a given test. The period of this steady region is defined by evaluating the mean HTC values over progressively longer time periods. Selecting the longest period before the mean starts to drive allows for the most accurate measurement.

The mean HTC value for the test displayed in Figure 7 produced in this way is $3.16 \pm 0.9 \times 10^{2} \mathrm{~W} / \mathrm{m}^{2} \mathrm{~K}$. The large uncertainty bounds highlight the difficulty in measuring exact heat transfer coefficients.

\section{Comparison of Nusselt Correlations}

Figure 8 compares Nusselt numbers calculated from data in Figure 7 and other tests analysed using the same method, plotted against effective Reynolds number. Tests were performed at four axial Reynolds number settings. The figure shows the correlations developed by Jakoby, Kim, and Wittig (1998) and Childs and Turner (1994) and the correlation developed in this study using the data collected for axial Reynolds number, $R e_{a}=4500$. The estimated uncertainty shown in the figure, \pm 16.2 , was taken from the test presented in Figure 7, which corresponds to $R e_{a}=4550$ and $R e_{\text {eff }}=$ $3.37 \times 10^{4}$. It is assumed that this is representative for all the experimental data points included in the graph.

A correlation was developed for an axial Reynolds number of $4.5 \times 10^{3}$, spanning an effective Reynolds number range of $4.6 \times 10^{3}$ to $3.4 \times 10^{4}$, corresponding to a Taylor number range of 0.0 to $9.0 \times 10^{8}$. The correlation is displayed in eq. (15) and has a $95 \%$ confidence interval of \pm 8.9 on the measured Nusselt values. 


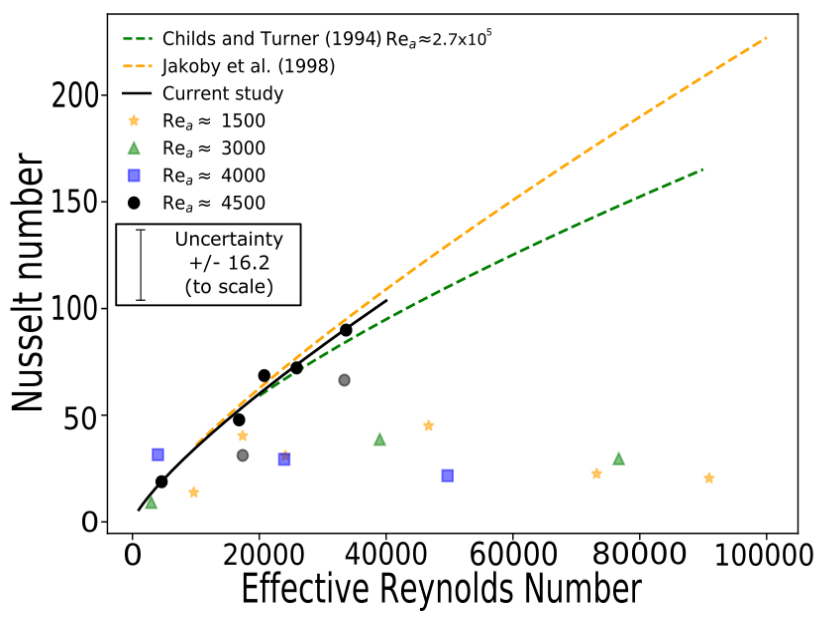

Figure 8 Comparison of Nusselt correlations for current study $\left(R e_{\text {eff }} \approx 4500\right)$ and Childs and Tuner (1994) and Jakoby et al. (1998). Displayed with data of other tests performed at different axial Reynolds numbers.

$$
N u=\frac{0.000185}{D_{h}} R e_{e f f}^{0.783}
$$

The correlation was developed discounting the two outlier points (shaded), as in these cases the HTC traces did not show a stabilised region, indicating different flow phenomena during these tests. The correlations developed by Jakoby, Kim, and Wittig (1998) and Childs and Turner (1994) show significant differences with each other, especially at higher effective Reynolds numbers. The correlation developed in this study lies between the other two correlations. These differences are indicative of the complexity of the T-C-P flow heat transfer process and that the process is affected by phenomena that are not captured by the two non-dimensional parameters, Nusselt number and effective Reynolds number, alone.

Data displayed in Figure 8 for lower axial Reynolds numbers, show a different trend. All data points exhibit a much lower Nusselt number, and after an initial increase, they appear to decrease with increasing effective Reynolds number. The clear differentiation between the low axial Reynolds number data $\left(R e_{a} \leq 4000\right)$ indicates a change in flow regime.

It is well known that flow structures and more turbulent flow regimes effect heat transfer. In purely axial annular flow, the transition to a turbulent flow regime occurs at $R e_{a} \approx$ 3000. Meanwhile, it is known that rotation tends to stabilise the flow, changing when transition occurs. This is consistent with the trend observed in the current study that shows a transition phenomena occurring at $R e_{a}>4000$, which leads to the observed increase in heat transfer, implying that a different, lower heat transfer regime exists for operating points at lower axial Reynolds numbers.

\section{CONCLUSIONS AND FUTURE WORK}

A test rig design and data analysis methodology for gathering T-C-P heat transfer data with high Taylor number flows is presented. A non-invasive methodology for determining heat flux is demonstrated with an in-depth uncertainty analysis using the Monte-Carlo method.

The full analysis of an example data set, corresponding to a Taylor number of $8.9 \times 10^{7}$ is presented. Analysis of the data highlight notable fluctuations in the mean measured HTC. A systematic analysis of measurement uncertainty and data analysis method yield corresponding uncertainty margins of the order $+/-0.9 \times 10^{2} \mathrm{~W} / \mathrm{m}^{2} \mathrm{~K}$. To the authors knowledge, this body of work represents the most in-depth analysis of the uncertainty in heat transfer for T-C-P flow regimes, highlighting the need for further work to improve measurement techniques.

The collected data for Taylor numbers range of 0.0 to $9.0 \times 10^{8}$ and effective Reynolds number range of $4.6 \times 10^{3}$ to $9.1 \times 10^{4}$, show that there are two distinct flow and heat transfer regimes separated by a critical value of axial Reynolds number. The critical value for axial Reynolds number is expected to lie between 4000 and 4500 . For the data with axial Reynolds number of 4500 , a correlation was created which compares favourably with prior correlations from literature. The resulting correlation with a $95 \%$ confidence interval of \pm 8.9 to the experimental data is:

$$
N u=\frac{0.000185}{D_{h}} R e_{e f f}^{0.783}
$$

Future works will include developing a correlation at higher Taylor number values. The presented rig design enables the use of high pressure and temperature fluids at high rotational speeds, allowing Taylor numbers up to $1 \times 10^{12}$ to be achieved.

\section{References}

Aoki, Hiroshi, Hidetaka Nohira, and Hajime Arai. 1967. "Convective Heat Transfer in an Annulus with an Inner Rotating Cylinder." Bulletin of JSME 10 (39): 523-32. https://doi.org/10.1299/jsme1958.10.523.

Ball, K.S., B. Farouk, and V.C. Dixit. 1989. “An Experimental Study of Heat Transfer in a Vertical Annulus with a Rotating Inner Cylinder." International Journal of Heat and Mass Transfer 32 (8): 1517-27. https://doi.org/10.1016/0017-9310(89)90073-2.

Battaglia, J. -L., O. Cois, L. Puigsegur, and A. Oustaloup. 2001. "Solving an Inverse Heat Conduction Problem Using a Non-Integer Identified Model." International Journal of Heat and Mass Transfer 44 (14): 2671-80. https://doi.org/10.1016/S0017-9310(00)00310-0.

Childs, P R N, and C A Long. 1996. "A Review of Forced Convective Heat Transfer in Stationary and Rotating Annuli." Proceedings of the Institution of Mechanical Engineers, Part C: Journal of Mechanical Engineering Science $\quad 210 \quad$ (2): 123-34. https://doi.org/10.1243/PIME_PROC_1996_210_179_02.

Childs, PRN, and AB Turner. 1994. "Heat Transfer on the Surface of a Cylinder Rotating in an Annulus at High Axial and Rotational Reynolds Numbers." In INSTITUTION OF CHEMICAL ENGINEERS SYMPOSIUM SERIES, 135:13-13. HEMSPHERE PUBLISHING CORPORATION. 
Dawood, H. K., H. A. Mohammed, Nor Azwadi Che Sidik, K. M. Munisamy, and M. A. Wahid. 2015. "Forced, Natural and Mixed-Convection Heat Transfer and Fluid Flow in Annulus: A Review." International Communications in Heat and Mass Transfer 62 (March): 45-57. https://doi.org/10.1016/j.icheatmasstransfer.2015.01.006.

Fénot, M., Y. Bertin, E. Dorignac, and G. Lalizel. 2011. "A Review of Heat Transfer between Concentric Rotating Cylinders with or without Axial Flow." International Journal of Thermal Sciences 50 (7): 1138-55. https://doi.org/10.1016/j.ijthermalsci.2011.02.013.

Gardarein, Jean-Laurent, Jean-Luc Battaglia, and Stefan Löhle. 2009. "Heat Flux Sensor Calibration Using Noninteger System Identification: Theory, Experiment, and Error Analysis." Review of Scientific Instruments 80 (2): 025103. https://doi.org/10.1063/1.3079328.

Heshmat, Hooshang, James F Walton $\mathrm{Ii}$, and José Luis Córdova. 2018. "Technology Readiness of 5th and 6th Generation Compliant Foil Bearing for 10 MWE S-CO2 Turbomachinery Systems," March, 29.

Howey, D. A., P. R. N. Childs, and A. S. Holmes. 2012. "AirGap Convection in Rotating Electrical Machines." IEEE Transactions on Industrial Electronics 59 (3): 1367-75. https://doi.org/10.1109/TIE.2010.2100337.

Jakoby, Ralf, Soksik Kim, and Sigmar Wittig. 1998. "Correlations of the Convective Heat Transfer in Annular Channels With Rotating Inner Cylinder." In Volume 4: Heat Transfer; Electric Power; Industrial and Cogeneration, V004T09A016. Stockholm, Sweden: ASME. https://doi.org/10.1115/98-GT-097.

Keep, Joshua A., Adam J. Head, and Ingo H. Jahn. 2017. "Design of an Efficient Space Constrained Diffuser for Supercritical CO 2 Turbines." Journal of Physics: Conference Series $821 \quad$ (1): 012026. https://doi.org/10.1088/1742-6596/821/1/012026.

Masuda, Hayato, Saho Yoshida, Takafumi Horie, Naoto Ohmura, and Makoto Shimoyamada. 2018. "Flow Dynamics in Taylor-Couette Flow Reactor with Axial Distribution of Temperature." AIChE Journal 64 (3): 1075-82. https://doi.org/10.1002/aic.15972.

Seghir-Ouali, S., D. Saury, S. Harmand, O. Phillipart, and D. Laloy. 2006. "Convective Heat Transfer inside a Rotating Cylinder with an Axial Air Flow." International Journal of Thermal Sciences $45 \quad$ (12): 1166-78. https://doi.org/10.1016/j.ijthermalsci.2006.01.017.

Smith, G. P., and A. A. Townsend. 1982. "Turbulent Couette Flow between Concentric Cylinders at Large Taylor Numbers." Journal of Fluid Mechanics 123: 187-217.

TACHIBANA, Fujio, Sukeo FUKUI, and Hisao MITSUMURA. 1960. "Heat Transfer in an Annulus with an Inner Rotating Cylinder.” Bulletin of JSME 3 (9): 11923. https://doi.org/10.1299/jsme1958.3.119.

Yamada, Yutaka. 1962. "Resistance of a Flow through an Annulus with an Inner Rotating Cylinder." Bulletin of $\begin{array}{llll}\text { JSME } & 5 & \text { (18): } & \end{array}$ https://doi.org/10.1299/jsme1958.5.302.

Yin, Feijia, Floris S. Tiemstra, and Arvind G. Rao. 2018. "Development of a Flexible Turbine Cooling Prediction Tool for Preliminary Design of Gas Turbines.” Journal of
Engineering for Gas Turbines and Power 140 (9): 091201. https://doi.org/10.1115/1.4039732.

\section{NOMENCLATURE}

$\begin{array}{cl}\text { Variables } \\ v & \text { Kinematic viscosity }\left(\mathrm{m}^{2} / \mathrm{s}\right) \\ \rho & \text { Density }\left(\mathrm{kg} / \mathrm{m}^{3}\right) \\ \mathrm{D}_{\mathrm{h}} & \text { Hydraulic diameter }(\mathrm{m}) \\ \mathrm{Ta} & \text { Taylor number } \\ \mathrm{Re} & \text { Reynolds number } \\ \mathrm{C} & \text { Coefficient } \\ \mathrm{V} & \text { Velocity }(\mathrm{m} / \mathrm{s}) \\ \mathrm{R} & \text { Radius }(\mathrm{m}) \\ \mathrm{r} & \text { Radius }(\mathrm{m}) \\ \omega & \text { Shaft angular speed }(\mathrm{rad} / \mathrm{s}) \\ \mathrm{q} & \text { Heat flux }\left(\mathrm{W} / \mathrm{m}^{2}\right) \\ \mathrm{Q} & \text { Heat flux matrix } \\ \mathrm{h} & \text { Convective heat transfer coefficient }\left(\mathrm{W} / \mathrm{m}^{2} \mathrm{~K}\right) \\ \mathrm{T} & \text { Temperature }(\mathrm{K}) \\ \mathrm{I} & \text { Impulse matrix } \\ \lambda & \text { Eigen value } \\ \alpha & \text { Thermal diffusivity }\left(\mathrm{k} / \rho \mathrm{p}_{\mathrm{p}}\right) \\ \mathrm{k} & \text { Thermal conductivity }\left(\mathrm{W} / \mathrm{mK}^{-1}\right) \\ \mathrm{t} & \text { Time }(\mathrm{s}) \\ \mathrm{J} & \text { Bessel Function of the First Kind }\end{array}$

\section{Subscripts/Superscripts}

$\begin{array}{cl}\mathrm{a} & \text { Axial component } \\ \mathrm{i} & \text { Interval value } \\ \mathrm{n} & \text { Eigen function } \\ \mathrm{N} & \text { Total number of time intervals } \\ \text { inner } & \text { Inner value } \\ \text { init } & \text { Initial value } \\ \phi & \text { Rotational component } \\ \mathrm{s} & \text { Surface } \\ \mathrm{f} & \text { Fluid } \\ \text { eff } & \text { Effective Value } \\ 0 & \text { Zeroth Order } \\ 1 & \text { First Order }\end{array}$

\section{ACKNOWLEDGMENTS}

I would like to acknowledge the Australian Solar Thermal Research Institute (ASTRI), and the Renewable Energy and Conversion Centre of Excellence (RECCE), The University of Queensland and the Australian Government for their financial support. I would also like to acknowledge my friends and family for their support through the process of doing this research. 